\title{
Adapting total quality management for general practice: evaluation of a programme
}

\author{
Martin Lawrence, Tim Packwood
}

\begin{abstract}
Objective - Assessment of the benefits and limitations of a quality improvement programme based on total quality management principles in general practice over a period of one year (October 1993 - 4).
\end{abstract}

Design - Questionnaires to practice team members before any intervention and after one year. Three progress reports completed by facilitators at four month intervals. Semistructured interviews with a sample of staff from each practice towards the end of the year.

Setting - 18 self selected practices from across the former Oxford Region. Three members of each practice received an initial residential course and three one day seminars during the year. Each practice was supported by a facilitator from their Medical Audit Advisory Group.

Measures - Extent of understanding and implementation of quality improvement methodology. Number, completeness, and evaluation of quality improvement projects. Practice team members' attitudes to and involvement in team working and quality improvement.

Results - 16 of the 18 practices succeeded in implementing the quality improvement methods. 48 initiatives were considered and staff involvement was broad. Practice members showed increased involvement in, and appreciation of, strategic planning and team working, and satisfaction from improved patient services. 11 of the practices intend to continue with the methodology. The commonest barrier expressed was time.

Conclusion - Quality improvement programmes based on total quality management principles produce beneficial changes in service delivery and team working in most general practices. It is incompatible with traditional doctor centred practice. The methodology needs to be adapted for primary care to avoid quality improvement being seen as separate from routine activity, and to save time.

(Quality in Health Care 1996;5:151-158)

Keywords: total quality management; general practice

Correspondence to:

Dr Martin Lawrence,

University of Oxford

Department of Public Health

and Primary Care, Gibson

Building, Radcliffe Infirmary,

Oxford OX2 6HE

Accepted for publication 28 June 1996 showing overall service improvement primary care. ${ }^{12}$ This is largely because tends to be topic based, retrospective, and usually involves few members of the primary healthcare teams. ${ }^{23}$ Total quality management seems to offer a more comprehensive approach to improvement, emphasising the need to set an overall strategy, concentrating on satisfying the needs of the customer or patient, developing improvement projects based on need, involving all members of the team, with the use of tools (only one of which is audit) to achieve improvement. In 1990 each of the 95 family health service authorities in England and Wales were required to establish a Medical Audit Advisory Group to support and monitor audit in the practices for which they were responsible. ${ }^{4}$ The groups usually appointed full or part time facilitators to work on audit with general practice teams. In 1993 the four Medical Audit Advisory Groups of the old Oxford Region together agreed to introduce and support a quality improvement programme for a group of practices, adapting total quality management methodology as appropriate for general practice. The programme was funded by the Department of Health, which required emphasis to be placed both on audit and Health of the nation topics. ${ }^{5}$

The programme was based heavily on the approach to quality improvement developed by Deming, ${ }^{67}$ and adapted for health care by the Institute of Health Care Improvement in Boston, Massachusetts. ${ }^{8}$ The three key elements to quality improvement emphasised are: for the practice leaders to set the strategy taking into account needs of both patients and the practice; for the practice to establish a culture for quality improvement with emphasis on communication and team working; and then for small multidisciplinary teams to use specific tools to carry out quality improvement projects.

We recommended that each participating practice establish a team of key people from each discipline (the quality executive) to manage the quality improvement programme. In consultation with the partners and practice members they were to agree strategy, select the projects, and arrange resources. For each project they set up and briefed a quality improvement team with at least one repesentative of each involved discipline, and supported and monitored the teams (fig 1).

The approach recommended for the project was Juran's solution (the Juran journey, fig 2) ${ }^{89}$ by which each small multidisciplinary quality improvement team defined its problem, examined this problem in more detail and considered possible solutions to identify the best, introduced that solution, and evaluated the change. The aim was to enable practices to develop a focus on quality, committed 


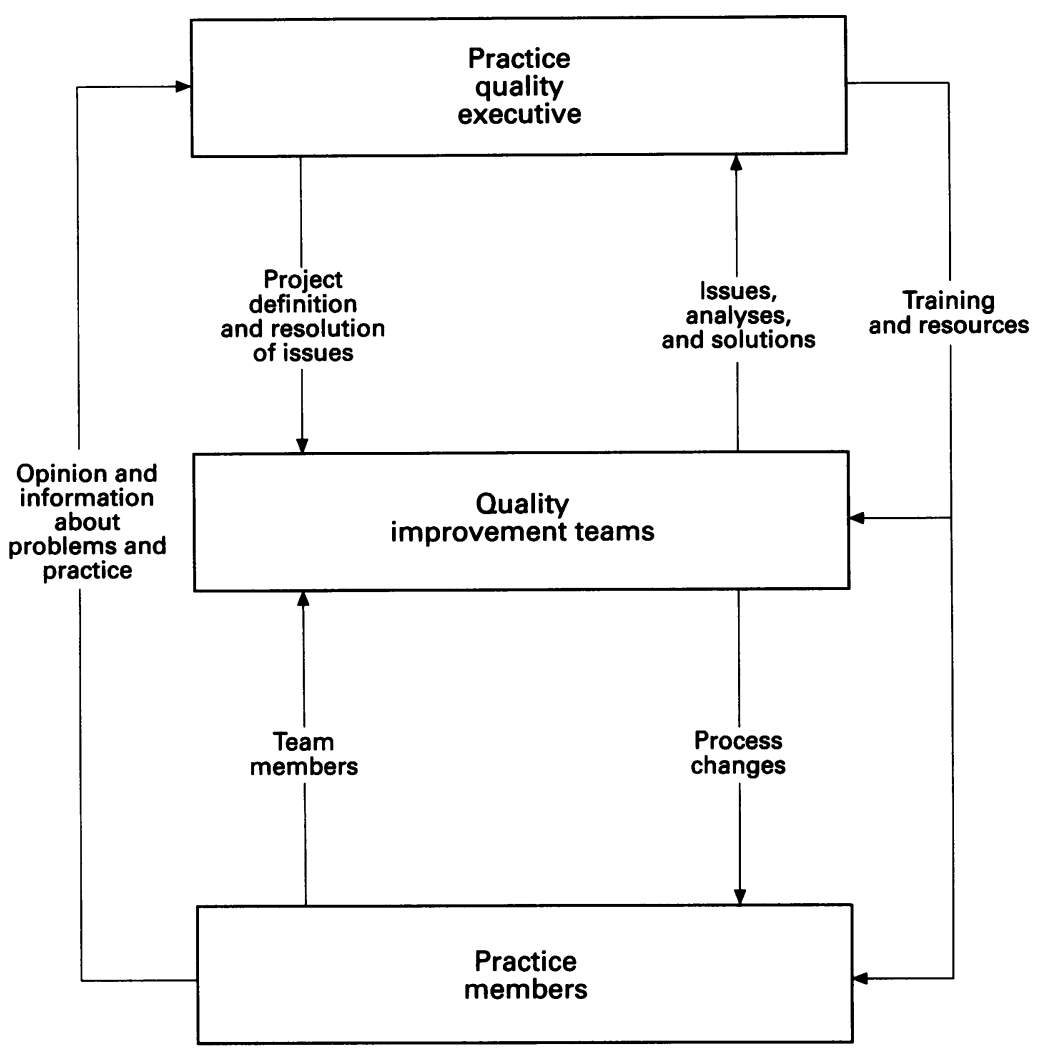

Figure 1 Organisation of a practice for quality improvement.

leadership, patient focus, team working, process based care, consideration of the evidence, continuous improvement, innovation, and proactivity. ${ }^{8}$

This paper briefly explains how the quality improvement programme was implemented, the mode of evaluation, and the major results. It concludes by discussing the strengths and weaknesses of the approach and the particular problems posed by primary care.

\section{Methods}

METHODS OF IMPLEMENTING QUALITY IMPROVEMENT

All the practices (360) in the old Oxford Region were invited by letter from the programme management committee to take part in a course and practice project for

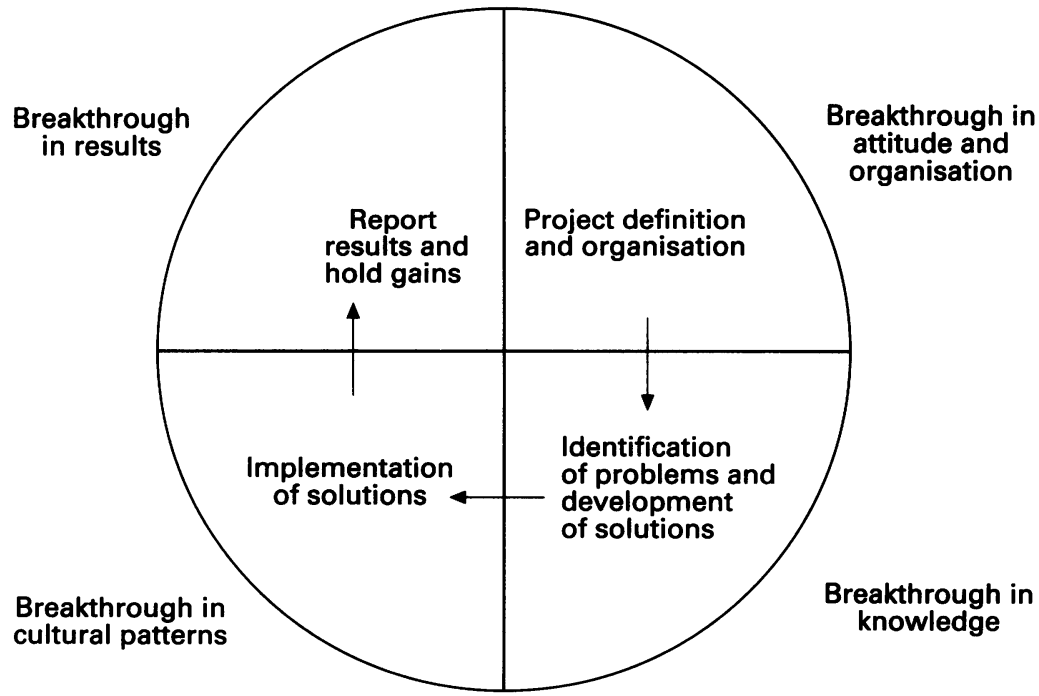

Figure 2 Implementation of the project: based on furan's solution. improving healthcare quality by total quality management which would last one year. They were told that three practice members of different disciplines would be offered a two day residential course and would be expected to introduce the methods to the rest of the practice; that they would receive support from a Medical Audit Advisory Group facilitator; that there would be follow up meetings during the year; that the practice would be expected to undertake at least two quality improvement projects; and that up to $£ 2500$ would be claimable for service and educational costs and expenses, justified by reports at successive stages of the programme.

The residential course was held in October 1993, with a member of the Institute of Health Care Improvement, Boston, Massachusetts, as an external advisor. Box 1 shows the key topics in the course. Each trio of practice members worked as a group, usually together with their facilitator, to model the introduction of quality improvement to their practices. All received a detailed course handbook which they were able to continue to use as reference. After the residential course, the practice representatives were expected to introduce the remainder of the practice team to the methodology, and to take the lead in its implementation.

Practices could undertake as many quality improvement projects as they wished, but two were expected to relate to areas identified as priorities in the government's Health of the nation initiative (coronary heart disease and stroke; cancers; mental illness; HIV/AIDS and sexual health; accidents). ${ }^{5}$ They were given a timetable for the year suggesting that strategy should be set and topics identified within three months; the identication of problems and development of solutions completed by six months; the implementation of solutions by nine months; and the report of results written up within the year.

Further support was provided by three workshops held at three monthly intervals, at which practice representatives exchanged and discussed progress and problems and received further advice, training, and support. Also the facilitators and management committee held five half day meetings to develop their own support and training.

METHODS OF EVALUATION

The Management Committee commissioned the Centre for the Evaluation of Public Policy

What is total quality management?

Principles of total quality management Focus on leadership, patients, process, teamwork

- Planning and developing practice strategy Including nominal group technique

- Problem solving methods Juran's solution (fig 2) Tools (brainstorm, flow charts, fishbone diagrams)

- Assessing patients' needs

- Organising the practice (fig 1)

- Managing change

Box 1 Key topics covered on the initial total quality management course for participating practices 
and Practice from Brunel University to undertake independent external evaluation of the programme. The particular areas for evaluation were: how participating practices implemented the process of quality improvement - what was done, by whom, and at what cost; if and to what extent the behaviour and attitudes of practice members changed about leadership, team working, and involvement in practice planning and quality improvement; the extent to which patients were involved in the quality improvement projects; the costs and benefits of this type of quality improvement programme; and its effects on work in the practice and on patients' care.

Methods were used to provide both quantitative and qualitative data as follows:

- Two questionnaires, both distributed by practice managers in the participating practices to all the doctors and attached staff at the beginning (October 1993) and end (October 1994) of the experimental period. One was a standard audit system questionnaire, produced by the Eli Lilly National Clinical Audit Centre; ${ }^{10}$ the second was designed for the project by the management committee. Box 2 shows the content of the two questionnaires. The responses were anonymous but respondents indicated their role in the practice, and the practices were requested to submit a list of their team members. All responses completed and returned to the evaluators were put on a database and the frequencies computed and compared.

- A process report designed by the evaluators which was completed for each participating practice by the associated Medical Audit Advisory Group facilitator on three occasions, in January, May, and October 1994. Box 3 shows the structure of the process

\section{First questionnaire:}

Do you understand audit?

Six point Likert scale between "no understanding" and "sufficient understanding to complete audit cycle"

Is there a practice policy on audit?

Choose one of five statements between "no policy" and "practice policy agreed and always applied"

Management of audit

Five point Likert scale to indicate level of agreement or disagreement with eight statements on the practice's management of audit

What value do you place on audit? $\mathrm{Six}$ point Likert scale between "audit is irrelevant" and "audit is essential".

How do you feel about audit?

Six point Likert scale on each of: enthusiasm; ability; involvement; and confidence

\section{Second questionnaire:}

Provide a written opinion on:

Does the practice work as a team?

What opportunities are there for team

working?

Can you express your views and do they count?

Do you know the practice's long term strategy?

Do you feel involved in planning future

direction?

Are there procedures for staff involvement in planning?
Practices were required to indicate the number and nature of projects being currently undertaken. Then, for each initiative:

- Date started

- Membership of quality improvement team

- Number of meetings held

Subject

People involved and their roles

Time taken

TQM techniques used

Outcomes

- Information collected

Nature

How collected

Time taken

Whether computerised

Whether analysed and by whom

Time taken for analysis

- Effects of initiative

On the practice

On patient care

- General comments on the process

Box 3 Content of process report forms

report forms. They provided a cumulative record of progress within each practice, as well as a source of comparative data between practices. The evaluators analysed responses manually by practice and by topic.

- Confidential, semistructured interviews. These were conducted by the evaluators towards the end of the project year, in the summer and autumn of 1994. Between three and six members of each practice were interviewed. In all cases the sample included at least one partner and one practice team member who had not been involved in a quality improvement team. Wherever possible the Medical Audit Advisory Group facilitators for each practice were also interviewed. Box 4 shows the interview schedule. Responses to the interviews were recorded by the evaluators on interview forms and analysed manually by practice and topic.

The three different sets of data were used in different ways.

The questionnaires were analysed by practice and by practice discipline to learn how respondents perceived their opportunities for collective working and participating in quality improvement processes, such as audit. The process reports were analysed by practice to give an indication of what was involved by participation in the programme, and details of the improvement projects undertaken. The third element, the interviews, enabled the evaluators to explore in more detail what the practice members thought had been achieved by the programme. Information from all three sources are presented in the results.

What has been accomplished by the quality improvement programme?

Has the approach led to effects on:

The work of individual people?

Team working?

Patients?

Was the approach significantly different from previous styles of working?

What have been the costs and benefits of quality improvement?

What is your overall view of the approach used in the quality improvement programme? 


\section{Results}

Nineteen practices enrolled in the programme and three members from each attended the course. Fourteen facilitators (five were responsible for two practices each), and five other members of the management committee also attended. One practice, which has been omitted from the analysis, withdrew at an early stage because they had recently begun to work with a management consultant and considered that the agendas might conflict. Eleven of the practices were fundholding.

Table 1 shows the numbers of questionnaires returned from before and after the programme. Because of confidentiality the anonymous replies could not be matched individually, but were classified by role. In calculating response rate the practice size was assumed to be the same at the end as at the start of the programme. One of the two practices which failed to implement total quality management did not complete the questionnaire at the end of the programme: it listed 18 members who were omitted from the 1994 calculations.

\section{QUALITY IMPROVEMENT PROJECTS}

Forty eight practice projects were undertaken of which 11 were completed by January 1995, with work continuing on 30 and seven postponed or abandoned. Two practices failed to develop strategies or complete projects. Three practices undertook only one subject, and one did as many as five. Six practices restricted themselves to the Health of the nation topics, three just worked at topics on practice organisation, and nine chose topics from both categories. Prevention of heart disease was the most popular of the Health of the nation topics, and appointment systems of the practice organisation topics, but projects varied from reducing the delay in doctors getting to the treatment room when requested by the practice nurses, to reorganising anticoagulation services within the practice, or understanding and overcoming patients' resistance to cervical cytology.

The process reports indicate that staff involvement was broad, with 265 members of the practices being recorded as involved over the year, receptionists (75) being the largest category. Six practices involved less than 10 staff and five more than 19; the numbers reflected the size of the practice. In all, 257 meetings were recorded; a mean (range) of 14 (4-27) a practice. The mean (range) meeting time was $19(3.5-51.25)$ hours a practice. Undoubtedly there were further time costs such as in collecting data, recorded as taking 103 hours of staff time in one practice.

\section{Examples of quality improvement projects}

Doctor availability to the practice nurse Problem: when the practice nurse required a doctor's attendance in the treatment room the delay seemed unreasonable.

Project team: general practitioner, practice nurse, receptionist, practice manager.

Identification of the problem and development of the solution: current procedure exam- ined (flow chart); different procedures reviewed and audited (mean (range) delay 6.5 (2-16) minutes); calling doctor directly quicker than through message desk; duty doctor quicker than usual doctor).

Implementation of solution: best procedure agreed (duty doctor through message desk); message receptionist trained; nurse protocols improved (reducing need for doctor); doctors trained to attend promptly.

Report of results: reaudit showed mean delay 2.8 minutes; a written protocol was introduced and discussed at a full practice meeting.

Secondary prevention of myocardial infarction Problems: (a) patients' needs may not be being met after myocardial infarction or coronary artery bypass surgery; (b) new evidence on benefits of secondary prevention may not be being implemented.

Project team: general practitioner; practice nurse; health visitor; receptionist; records clerk.

Identification of the problem and development of the solution: focus group with patients; flow chart of present procedures; need identified for early home visit, local rehabilitation services, and follow up clinic at surgery.

Implementation of solution: first day visit to be made by general practitioner or health visitor; exercise sessions arranged at local gym; nurse run follow up clinic implemented.

Report of results: audit (at nine months) shows that $20 / 78$ patients attend the gym, $69 / 78$ have attended the nurse clinic.

Reducing errors in repeat prescribing - Problem: too many errors in the writing or timely production of repeat prescriptions.

Project team: general practitoner; practice manager; practice nurse; receptionist; pharmacist.

Identification of the problem and development of the solution: "fishbone diagram" of all procedural problems.

Implementation of solution: each problem discussed and remedied

Report of results: repeat two week audit showed errors or delays reduced from 26 to 11 .

\section{PRACTICE PLANNING AND STRATEGY}

At the start of the programme most practices did not have a long term strategy, and if they did most staff were usually unaware of it. All the practices had prolonged planning meetings or away days as part of the programme, and this was new to most practices, as were group decision making processes. None of the practices claimed to have completely dispensed with hierarchy, but some made a considerable movement towards democratic working processes.

For example, in one practice all the GPs but no other health professionals considered themselves involved in planning at the outset; by the time of the second questionnaire survey half the health professionals felt involved, and the practice manager was aware of long term strategy. In another practice a practice manager moved from "not usually consulted" to "yes, 
very much so". Clerical staff were less involved, but remarks at interview included "previously we lurched from crisis to crisis, now we are being proactive and scientific"; "we always knew there were lots of resources in the practice we were not using"; "primary care team members feel their problems are being addressed, now or in the future".

\section{Example of practice planning}

A practice that had never involved its staff in strategic planning held an "away day" as part of the programme. In advance they asked each discipline to hold a meeting and decide priorities - all put communications near the top except the doctors who put it 12th on their list. After the away day - which did find some tensions and required expert help - one of the procedures agreed to improve communication was a project led by the practice nurse to improve the nurse clinic which was joint between the three practices in the health centre. The practices had never cooperated before. The project was so successful that the practices are now working jointly on a system for visiting the bereaved.

\section{LEADERSHIP}

The development of a quality executive enabled leadership to be shared, but the process reports showed that doctors were involved in the leadership of 31 projects. Interview responses indicated that the doctors' involvement was seen as crucial and symbolic of their commitment to change. Practice managers led or co-led 15 projects, and health visitors and practice nurses also led projects.

As important as leadership was the potential wrecking power of traditional practice leaders. It was noticeable how practices with partners who were said at interview to have obstructed or negated initiatives, achieved little. Two practices achieved neither a strategy nor completed projects; in one the doctors could not agree on any joint project, in the other the senior partner refused to implement any of the proposals of the project team. Incorporation of general practitioners and practice managers into teams was seen as having a very positive effect - "it's good for doctors who are used to being autocratic and making decisions without involving others".

\section{Example of leadership development}

In one practice the practice manager tended to handle issues with little consultation, and the general practitioner who attended the course was near retirement. The programme started well in the practice, but they had difficulty getting teams to progress - indeed they thought that they were failing and almost gave up. But one project, on asthma care by a team led by a health visitor, went well. Audit showed improved care, the patients were appreciative, and the experience changed the attitudes of many practice members. The practice manager now leads with consultation, the doctors seek information before taking decisions, and the staff are used to getting together to analyse and solve problems for themselves.

\section{TEAMWORK}

Different practices started with different levels of teamwork, but the facilitators interviewed thought that one of the most important changes was the way that practices were working more as teams and that there had been a degree of change in most practices. Remarks by team members included: "the atmosphere is now more friendly and congenial"; "there is better understanding of the other disciplines, breaking down the them and us"; "it crosses the GP - staff divide which had been a problem"; "a personal bond between group members".

On the other hand an opening of team issues sometimes highlighted inadequacies and discontent previously accepted. This was especially the case with non-medical staff; "we pay lip service to teamwork"; "poor communication prevents teamwork"; "the doctors do not value us". And it was a problem when the practice had been very hierarchical and the doctors found it hard to be less directive; "expressing your opinion is difficult, they still pay your wages"; "the staff don't expect change in the directive nature of the lead partner".

\section{AUDIT}

Attitudes towards audit were assessed by the first questionnaire: not all respondents completed every section of the questionnaire. Among medical staff, 63\%(125/198) thought that they had a good understanding of audit at the beginning of the year, and $82 \%(113 / 138)$ by the year end; $81 \%(147 / 182)$ thought that audit was valuable at the start, and $89 \%(121 / 136)$ at the end; and $60 \%(96 / 161)$ began the year seeing audit as a normal part of practice, rising to $71 \%(95 / 133)$ by the year end. For non-medical staff at the beginning of the project $33 \%(49 / 147)$ understood audit, $77 \%(96 / 135)$ valued it, and $81 \%(87 / 108)$ saw audit as normal practice. By the end of the year $46 \%(60 / 131)$ understood it, $75 \%(80 / 106)$ valued it, and $72 \%(79 / 110)$ saw it as normal practice.

\section{INVOLVEMENT OF PATIENTS}

Involvement of the patients was reported in the process reports in 23 of the 48 projects. This varied from focus groups (convened to discuss patients' needs - for example, heart disease rehabilitation, or telephone access); questionnaires; interviews - for example, over access and information for cervical cytology; or representation on the Quality Improvement Team. Remarks at interview suggested an improved perception of patients' needs, particularly in the management of their care: "we now emphasise the perspective of the patients rather than "we know best" "; "we ensure that procedures are congruent with patients' priorities"; or, specifically, "transport is the biggest problem in reaching cardiac rehabilitation".

\section{BENEFITS AND COSTS}

Some estimate of benefits and costs was sought in both the process reports and the interviews. In only one practice were all the interviewees doubtful as to the value of the programme. 
Positive effects cited included staff satisfaction and culture change, such as more opportunities to contribute to practice planning; achievement of change, both by tackling problems and by using the best solutions; better working practices, as in the organisation of appointment systems and better use of staff time; greater awareness of patients' needs; and better systems of caring for particular categories of patient - such as teenagers or those with heart disease. Many also reported that the experience had been fun, enjoying the process of contributing.

The overwhelming cost mentioned was time, reported by every participating practice. Projects were seen as extra activities to be undertaken as well as usual work, a problem particularly for doctors and part time staff. The other major perceived cost was in opening up the issue of working relations - this was painful, especially for some doctors and practice managers who had been used to control.

\section{Discussion}

ACHIEVEMENTS OF THE QUALITY IMPROVEMENT PROGRAMME

The programme achieved change. Although two of the practices failed to develop projects at all, seven of the 18 practices completed projects, and a further six had them ongoing. Many practices held full practice meetings for the first time, and developed long term strategies within which to plan improvements. Issues of leadership were clarified, and some dysfunctional leaders were able to develop through team working. Most staff thought that there were improvements in team working, and although practices which already had a team oriented approach were most successful in completing projects, several achieved multidisciplinary working on improvement projects for the first time. Many practice team members said that they had enjoyed working with the programme, and thought that they were offering a better service for patients.

These benefits came directly from the total quality management approach, attending to the culture of the practice at the same time as working in depth together on projects to improve patient care. In a final survey 11 of the practices said that they intended to use some total quality management principles in future.

It was notable that although medical team members developed a better understanding and appreciation of audit, understanding improved in non-medical members but the valuing of audit worsened, perhaps because better understanding raised expectations.

PROBLEMS RELATING TO PRIMARY CARE

Primary care is not an easy setting within which to introduce a quality improvement project of this kind, for several reasons. Firstly, it is traditionally hierarchical, with the doctors as owner managers and the staff as employees, and the extent to which doctors incorporate staff in planning has been limited, as has been the freedom of staff to make changes. Secondly, doctors see themselves as gatekeepers and custodians of patients' health, and may be reluctant to see patients' perceived needs as relevant. These are crucial in quality improvement strategies. Thirdly, practices are often small, and have no surplus staff for dedicated quality activity. The programme took place when the 1990 contract was at its most demanding with many externally determined targets. ${ }^{11}$ Much of the energy of the practices was spent on the achievement of these tasks, directly contrary to the principles of quality improvement. Finally, there is no reward system in general practice for quality. Most financial return is for structural issues - such as the size of the list - or for achieving short term process goals - such as recording cervical smears. The incentives for producing quality services are therefore largely altruistic, together with the hope that effort invested in improving processes will produce long term efficiency savings - not an easy concept to convey to a hard pressed practice.

PROBLEMS RELATING TO THE PROGRAMME

There have been very few attempts to adapt total quality management to primary care, ${ }^{12}$ and the members of the management committee were learning as fast as the participants. Indeed, part way through the year a management consultant was recruited to give support and guidance to the management committee and facilitators. It was decided to use Medical Audit Advisory Group facilitators rather than a special facilitator for the programme, partly to save money and partly so that the expertise would remain in the region after the project ended. But these facilitators had to be trained as the initiative proceeded and inexperience often resulted in replacing the usual "just in time training" with "just too late training". Nevertheless, the practices expressed appreciation of their facilitators' support, which, depending on the needs of the practice and abilities of the facilitator, ranged from telephone contacts with occasional personal support, to periods of intensive work on a weekly or fortnightly basis.

There were constraints on the programme. Firstly, the requirement that two projects were on Health of the nation topics was an unnatural constraint for a quality improvement programme. Several practices ignored it and began with smaller scale organisational problems, and from this it was apparent that the successful completion of a small and clearly relevant project greatly improved practice members' attitudes to the approach.

A second constraint was the time scale. Quality improvement is usually regarded as a long term exercise. Authorities usually quote five years or more to produce significant change in the working of a business. ${ }^{613}$ Because of the short term funding of this particular initiative, the intervention was designed to run for 12 months with the evaluation completed within three months of the intervention ending. This is a short time scale within which to measure success.

PROBLEMS RELATING TO THE EVALUATION In retrospect the evaluation was both too ambitious, adding to the time costs of the par- 
ticipating practices, and too uncontrolled, in that questionnaires and process reports were not completed consistently between or within practices. In particular practices varied in the number of associated members (especially community nurses and health visitors) that they listed as belonging to their teams. These were the members whose rate of returning the questionnaire after the programme fell most sharply (table 1), which may reflect that they were least involved in the programme. These variations detracted from the reliability of the findings and their value for comparative purposes.

These problems accepted, the mixture of quantitative and qualitative approaches did succeed in providing rich insights into the way the quality improvement programme was applied and implemented within 18 very different general practices. The programme also suggested several practical lessons that might be considered in any similar programme.

\section{ADAPTING TOTAL QUALITY MANAGEMENT FOR GENERAL PRACTICE}

It is clear that methods typically used in quality improvement based on total quality management need to be adapted for general practice teams and concentrate on those that correlate closely with success.

Away days (or half days) were universally approved by practice team members, as a major advance in incorporating the whole practice into planning and in making the whole team aware of direction and priorities. Occasional team meetings during the year enabled communication and education to continue, but meetings within disciplines (nurses, receptionists, etc) were also important in developing work patterns.

The existence of a well functioning quality executive with at least one medical member led to success, partly because it ensured endorsement by the partners, partly in keeping programmes up to time, and partly in supporting any group attempting change and improvement.

Thereafter the message of the evaluation was "keep it simple". Early topics need to be small, achievable, and relevant; project teams should be small, containing each person essential to the topic to be examined, but no more; indeed affected team members can get together as quality improvement teams to discuss problems and explore situations whenever necessary; communication is helped by notice

Table 1 Team members listed by the participating practices, and questionnaires returned

\begin{tabular}{lllllllll}
\hline & $G P$ & $P N$ & $H V$ & $C N$ & $P M$ & $\operatorname{Rec}$ & Other & Total \\
\hline $\begin{array}{l}\text { Members (1993) } \\
\text { Questionnaires } \\
\text { returned (1993): }\end{array}$ & 89 & 56 & 39 & 82 & 21 & 136 & 74 & 497 \\
$\quad \begin{array}{l}\text { No } \\
\text { (\%) }\end{array}$ & 67 & 46 & 28 & 38 & 21 & 89 & 56 & 345 \\
$\begin{array}{l}\text { Questionnaires } \\
\text { returned (1994): }\end{array}$ & $(75)$ & $(82)$ & $(72)$ & $(46)$ & $(100)$ & $(65)$ & $(76)$ & $(69)$ \\
$\quad$ No & & & & & & & & \\
$(\%)$ & 57 & 31 & 18 & 22 & 20 & 79 & 45 & 272 \\
& $(67)$ & $(58)$ & $(49)$ & $(28)$ & $(95)$ & $(60)$ & $(61)$ & $(55)$ \\
\hline
\end{tabular}

$\mathrm{GP}=$ general practioner; $\mathrm{PN}=$ practice nurse; $\mathrm{HV}=$ health visitor; $\mathrm{CN}=$ community nurse; $\mathrm{PM}=$ practice manager $; \mathrm{Rec}=$ receptionist. boards in the staff areas recording progress, or by occasional meetings within disciplines; and beneficial change needs to be implemented with the minimum of fuss without the project team feeling constrained to go through all the steps set out by Juran before being allowed to make changes. Practices found that the major sources of data they required were in patient records or could readily be obtained by simple surveys or interview programmes.

Assessment of patients' needs has tended to be perceived as irrelevant and threatening. But practices who undertook it expressed benefit both in defining direction and providing satisfaction. Some methods, such as focus groups or random interviews, are neither threatening nor time consuming, and can sometimes be undertaken as well by non-medical as by medical staff.

Training is a major issue. There may be expertise within the practice, but in this initiative the Medical Audit Advisory Group facilitators were greatly valued. Developing alliances between Medical Audit Advisory Groups and postgraduate medical education may make this facility more relevant and accessible for practices. ${ }^{2}$

Finally Health Commissions have a major part to play. Already some are requesting practice development plans and education programmes, and this provides practices with an incentive to develop quality programmes, and to use some of the above techniques to achieve them.

\section{Conclusion}

Busy practices may regard a quality improvement programme as an unnecessary addition to their already overfilled agendas. But existing audit programmes will neither fully develop the potential of the practice for quality, nor enable all the team members to contribute fully. This programme showed how attention to the practice culture and organisation, at the same time as systematically working on improvement projects, can produce change and improve services and morale. Adaptation of total quality management methods can enable the system to be incorporated with little additional time into normal working.

The Quality Improvement Programme was funded by the NHS Management Executive under the Medical Audit Projects Programme: the management committee was chaired by Dr M gramme: the management committee was chaired by Dr $M$ Regional Audit Committee: it was undertaken by Tim Packwood, Valerie Beale, and Grant Franks. Figures 1 and 2 Packwood, Valerie Beale, and Grant Franks. Figures 1 and 2 "Improving Health Care Quality", copyright of the National Demonstration Project.

Humphrey C, Hughes J. Audit and development in primary care. London: King's Fund Centre, 1992.

2 Primary Health Care Clinical Audit Working Group of the Clinical Outcomes Group. Clinical audit in primary health care. London: Department of Health, 1995 .

3 Lawrence M, Griew K, Derry J, Anderson J, Humphreys J. Auditing audits: use and development of the Oxfordshire Auditing audits: use and development of the Oxfordshire Medical Audit Advisory Group rating system. BMF 1994;

4 Department of Health. Medical audit in the family practitioner services. London: DOH, 1990. (Health circular $\mathrm{HC}(\mathrm{FP})(90) 8$.

5 Secretary of State for Health. The health of the nation: a strategy for health in England. London: HMSO, 1991.

6 Deming WE. Out of the crisis. Massachusetts Institute of Technology, Cambridge, MA, 1986. 
7 Walton M. The Deming management method. London: Mercury, 1992

8 Institute for Health Care Improvement. Improving healt care quality; a comprehensive course. Boston: IHI, 1991.

9 Juran J. Furan on planning for quality. New York: Free Press, 1988

10 Hearnshaw H, Robertson N, Cornie R, Darling L, Baker R. Audit for teams project. Leicester: Lilly National Clinical
Audit Centre, 1993. (Research report No 1.)

11 Department of Health and Welsh Office. General practice in the National Health Service. A new contract. London HMSO, 1989.

12 Zairi M, Matthew A. TQM in primary care: an evaluation. Bradford: University of Bradford, European Centre for total quality management, 1993.

13 Crosby P. Quality is free. New York: McGraw Hill, 1979. 